\title{
Hybrid Error Control and Security Framework for Reliable Cornea and Infrared Video Communication in loT Applications
}

Walid El-Shafai ( $\nabla$ eng.waled.elshafai@gmail.com )

Menoufia University https://orcid.org/0000-0001-7509-2120

Amany Daosh

Menoufia University

Nehad Haggag

Menoufia University

Aya Gamal

Menoufia University

Nevien Sadik

Menoufia University

Yasser Mahrous

Menoufia University

Fatma Ibrahim

Menoufia University

Naglaa Soliman

Menoufia University

Abeer Algarni

Menoufia University

Ghada El Banby

Menoufia University

Mohamad R. Abdelrahman

Menoufia University

Said Eldosary

Menoufia University

Emad S. Hassan

Menoufia University

Huda Ashiba

Menoufia University

Eman Soltan

Menoufia University

Waleed Alhanafy 
Menoufia University

\section{Adel Saleeb}

Menoufia University

\section{Adel El-Fishawy}

Menoufia University

\section{S. El-Rabaie}

Menoufia University

\section{M. El-Halawany}

Menoufia University

Moawad I. Desouky

Menoufia University

\section{Sami El-Dolil}

Menoufia University

\section{Nabil Ismail}

Menoufia University

Ibrahim M. El-Dokany

Menoufia University

Fathi E. Abd El-Samie

Menoufia University

\section{Research Article}

Keywords: Cornea and infrared frames, B-SVD, SVD, BKF, Disparity and motion compensation, Quality-ofService, Wireless networks

Posted Date: March 22nd, 2021

DOI: https://doi.org/10.21203/rs.3.rs-276267/v1

License: (c) (1) This work is licensed under a Creative Commons Attribution 4.0 International License. Read Full License 


\title{
Hybrid Error Control and Security Framework for Reliable Cornea and Infrared Video Communication in IoT Applications
}

Walid El-Shafai, Amany Daosh, Nehad Haggag, Aya Gamal, Nevien Sadik, Yasser Mahrous, Fatma Ibrahim, Naglaa Soliman, Abeer Algarni, Ghada El Banby, Mohamad R. Abdelrahman, Said Eldosary, Emad S. Hassan, Huda Ashiba, Eman Soltan, Waleed Alhanafy, Adel Saleeb, Adel El-Fishawy, S. El-Rabaie, M. M. El-Halawany, Moawad I. Desouky, Sami El-Dolil, Nabil Ismail, Ibrahim M. El-Dokany, Fathi E. Abd El-Samie

Department of Electronics and Electrical Communications Engineering, Faculty of Electronic Engineering, Menoufia University, 32952 Menouf, Egypt

eng.waled.elshafai@gmail.com, amany.abdelaziz@te.eg,nehad.haggag@elarabyhospital.com, ayagamal@gmail.com, neviensedik@yahoo.com, yasser.mahrous@hotmail.com, fatmaibrahim@yahoo.com, naglaasoliman@gmail.com, abeeralgarni@gmail.com, ghababanby@yahoo.com, abdrahmanmohamed@yahoo.com, eldosrysaid@gmail.com, emadhassam@yahoo.com, hudaashiba@gmail.com, emansoltan@gmail.com, waleedhanafy@gmail.com, adelsaleb@yahoo.com, fishawyadel@yahoo.com, elsayedelrabaie@gmail.com,mmohamedelhalawany@gmail.com, moawadibrahim@gmail.com, samidolil@yahoo.com, nabilismail@gmail.com,dokanyibrahim@yahoo.com, fathi_sayed@yahoo.com

\begin{abstract}
In this paper, we present hybrid watermarking and error control techniques for reliable cornea and infrared frame communication through wireless networks in Internet of Things (IoT) applications. In the proposed watermarking technique, two stages of Singular Value Decomposition (SVD) watermarking are used. In the embedding stage, two watermark images are embedded in either the cornea or infrared frames using Block-based SVD (B-SVD) and SVD schemes, respectively. Then, the resulting watermarked cornea or infrared frames are transmitted through an erroneous wireless channel. At the receiver, the received corrupted cornea or infrared frames are recovered using a proposed hybrid post-processing error control technique. This technique comprises a Circular Spatial-scan Order Interpolation Algorithm (CSOIA) and a temporal Partitioning Motion Compensation Algorithm (PMCA) to reconstruct the erroneous cornea or infrared frames. Then, the Bayesian Kalman Filter (BKF) is utilized in the amelioration process due to its efficiency to smooth the remanent inherent corruptions in the formerly reconstructed frames to obtain high cornea or infrared video quality. After that, the watermark extraction stage is implemented to extract the watermark images from the watermarked frames. Simulation results on several cornea and infrared frames show that the suggested hybrid watermarking, and error control techniques reveal adequate subjective and objective video quality compared to those obtained with the traditional methods. In addition, the watermark detectability, robustness, and security are enhanced. Moreover, the experimental results show that the proposed watermarking technique is superior and more secure than the other previous techniques for embedding and extraction of watermarks, efficiently, in the presence of attacks.
\end{abstract}

Keywords Cornea and infrared frames, B-SVD, SVD, BKF, Disparity and motion compensation, Quality-of-Service, Wireless networks.

\section{Introduction}

Today, the most important way to connect people all over the world is the Internet. It allows users to communicate and share multimedia data such as text, image, audio, and video, with low cost and high quality. In addition, the emergence of IoT has led to the need to send multimedia content over IoT networks. So, recently, the research efforts in wireless multimedia communication have increased and received more attentiveness. The multimedia communication over wireless networks or Internet has increased, dramatically [1-6]. However, one of the most serious problems that arise during multimedia communication is how to protect the critical data during the transmission process from illegal use [7, 8]. Another serious problem of multimedia transmission over wireless networks or Internet is the induced errors on the communication channels [9-14]. Thus, to transmit the multimedia data over erroneous channels, efficient encoding must be implemented to maintain a good reception performance. Therefore, the video 
encoding prediction structure must depend on the time- and spatial-domain correlations inside frames to improve the decoding performance. Unfortunately, the highly-encoded videos are very sensitive to transmission channel errors.

Protecting confidential multimedia information is a moral and legal requirement. Data hiding is one of the protection ways in which part of the information is hidden into a multimedia object to authenticate it. This process is performed in such a way that the embedded data is not perceptible to an observer, but it is easily detected by the detector. Data hiding has two main categories: digital watermarking and steganography $[15,16]$. The insertion of a part of information into the multimedia content is called digital watermarking. This information should not be to a human eye, but can be detected with an appropriate algorithm. Figure 1 presents the general concept of the watermarking algorithm, which consists of the embedding and detection processes. This algorithm is suitable for several applications such as fingerprinting, copyright protection, and content authentication, because it has a very important feature that the content is inseparable from the watermark. The required characteristics of digital multimedia watermarking include imperceptibility, high robustness, high capacity, and large degree of security [17-19]. The general requirements for designing an efficient multimedia watermarking algorithm can be outlined as shown in Fig. 2 . They are defined as follows:

1. Imperceptibility. The watermarked signal should be invisible for the human eye. In other words, the original host signal or image should not be affected by the embedded data.

2. High robustness. The watermarked signal should resist modifications such as removal or elimination of embedded data by attacks from unauthorized persons.

3. High capacity. The amount of embedded data in the host signal or image should be large as much as possible.

4. Large degree of security. The authorized person is the only one, who can detect the watermark.

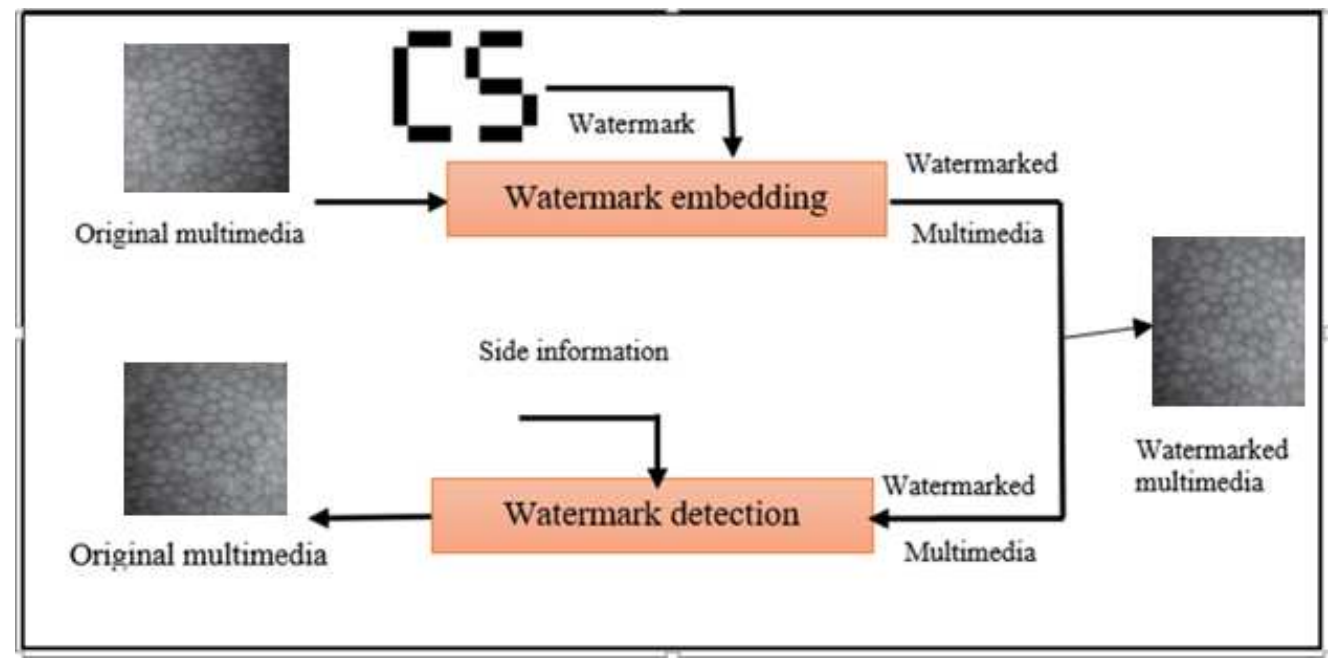

Fig. 1. Watermarking system for multimedia content.

The multimedia content such as medical corneal and infrared videos to be streamed over wireless channels is subject to random and bursty packet corruptions due to packet dropping or fading-motivated bit errors [20-23]. So, errors might propagate to the neighbouring and the subsequent frames, resulting in inefficient video quality. It is intractable to retransmit the corrupted or erroneous frames due to delay restrictions on real-time video applications. So, there is a need for error control techniques such as 
post-processing concealment techniques at the receiver side. The concealment techniques are recommended, as they decrease the channel errors without increasing the delay or requiring any complicated encoder adjustments [4, 9, 11].

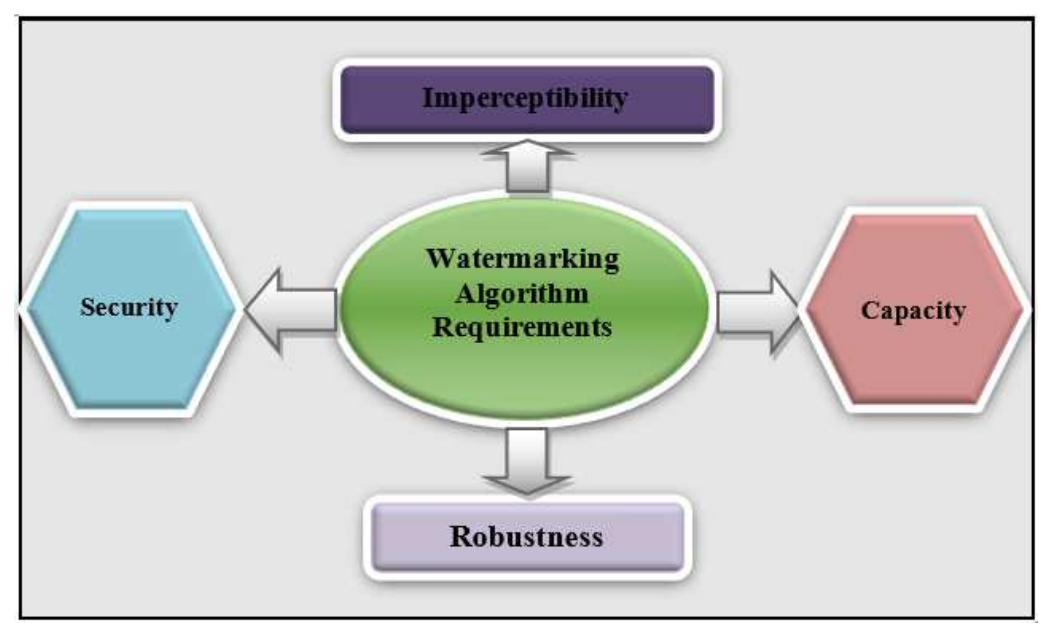

Fig. 2. Multimedia watermarking requirements.

The cornea is the transparent layer that faces the light, which enters the eye. Cornea and Sclera form the outer tunic of the eye and are mechanically strong. They protect the eye from any foreign object. The cornea has five main layers, namely epithelium, Bowman layer, Stroma, Descemet membrane, and endothelium. The main function of the endothelium is to pump the excess water out of the stroma to preserve its mechanical structure and optical clarity. The corneal endothelium has an important impact on the human vision because of the direct relationship between the healthy cornea and the endothelium cells [24-26]. In this paper, we will transmit and evaluate corneal frames over wireless networks for telemedicine applications. In addition, we will investigate also the transmission of infrared video frames over wireless networks for further analysis. Both types of video frames are considered in IoT applications.

Several error control techniques are suggested to minimize the effect of packet damages during the video transmission process. In the state-of-the-art works [27-29], the Automatic Repeat Request (ARQ) and Forward Error Correction (FEC) algorithms have been proposed. Unfortunately, they increase the transmission bit rate, and they are not recommended for real-time video applications. Therefore, in this paper, we propose efficient post-processing error control techniques at the decoder, which neither add redundant data nor increase the transmission bit rate. The proposed post-processing error control techniques in this work have the feature of enhancing the received video quality without any modifications in the encoder software or hardware or in the transmission bit rate. These techniques depend on the time and spatial correlations inside the corneal and infrared sequences to conceal the corrupted frames. At the decoder, a hybrid approach of CSOIA and PMCA is discussed in section 3.2 in order to determine the Disparity Vectors (DVs) and Motion Vectors (MVs) of the corrupted video frames. After that, for more enhancement of the concealed corneal and infrared video frames, the BKF is applied on the recovered DVs and MVs to enhance the overall video quality.

The main contributions of this paper are summarized as follows:

1. A hybrid framework comprising error control coding and watermarking is introduced for reliable transmission of corneal and infrared video frames over a wireless communication channel. 
2. The proposed hybrid framework achieves superior performance compared to the traditional algorithms in mitigating the effects of communication channel errors and multimedia attacks.

3. An efficient hybrid algorithm based on block-based SVD watermarking and traditional SVD watermarking is introduced. This hybrid algorithm improves the copyright protection against severe attacks.

4. The proposed hybrid watermarking algorithm improves the capacity by embedding two different watermarks without affecting the imperceptibility and visual quality of the watermarked frames. This algorithm achieves an acceptable performance of watermark detection compared to the traditional algorithms.

5. The proposed hybrid error control techniques have a great effect on improving the overall performance of the proposed framework by achieving superior performance in watermark extraction.

The rest of this paper is organized as follows. Section 2 introduces the related work and the basics of the SVD watermarking process. In section 3, we present the proposed hybrid watermarking algorithms, the proposed error control techniques for corneal and infrared frames, and the suggested BKF amelioration technique. Simulation outcomes and comparative discussions are summarized in section 4 . Section 5 presents the concluding remarks.

\section{Related Work}

\subsection{Traditional SVD Watermarking}

From the image processing perspective, the SVD is a mathematical technique which ensures good stability for Singular Values (SVs). When small changes occur in the watermarked image, its SVs do not change majorly. Hence, SVD watermarking is robust against different attacks. The algebraic properties of an image can be represented by the SVs of that image. Using these properties of the SVD of an image, the watermark can be embedded in the SV matrix without large variations in the watermarked image. Liu et al [30] introduced a watermarking algorithm based on the spatial-domain SVD. Recently, watermarking schemes based on SVD have gained popularity due to the very simple implementation process and the attractive mathematical features of the SVD. In the SVD, any matrix $\mathbf{B}$ may be written as [30]:

$$
\mathbf{B}=\mathbf{X S Y} \mathbf{Y}^{T}
$$

where $\mathbf{X}$ and $\mathbf{Y}$ are orthogonal matrices such that $\mathbf{X}^{T} \mathbf{X}=\mathbf{I}, \mathbf{Y}^{T} \mathbf{Y}=\mathbf{I}$, and $\mathbf{I}$ is an identity matrix. $\mathbf{S}$ is a diagonal matrix containing the SVs of the matrix $\mathbf{B}$. The columns of $\mathbf{X}$ are the left SVs of $\mathbf{B}$, and the columns of $\mathbf{Y}$ are the right SVs of $\mathbf{B}$. Figure 3 illustrates the watermark embedding process for the traditional method of Liu [30].

\subsubsection{Embedding Process:}

The steps of watermark embedding are as follows:

1. The original image ( $\mathbf{B}$ matrix) is decomposed with SVD.

$$
\mathbf{B}=\mathbf{X S} \mathbf{Y}^{T}
$$

2. The matrix $\mathbf{D}$ is obtained by adding the watermark ( $\mathbf{W}$ matrix) to the SVs of the original matrix.

$$
\mathbf{D}=\mathbf{S}+k \mathbf{W}
$$

3. The new modified matrix ( $\mathbf{D}$ matrix) is decomposed by SVD.

$$
\mathbf{D}=\mathbf{X}_{w} \mathbf{S}_{w} \mathbf{Y}_{w}^{T}
$$


4. The watermarked image is obtained as follows:

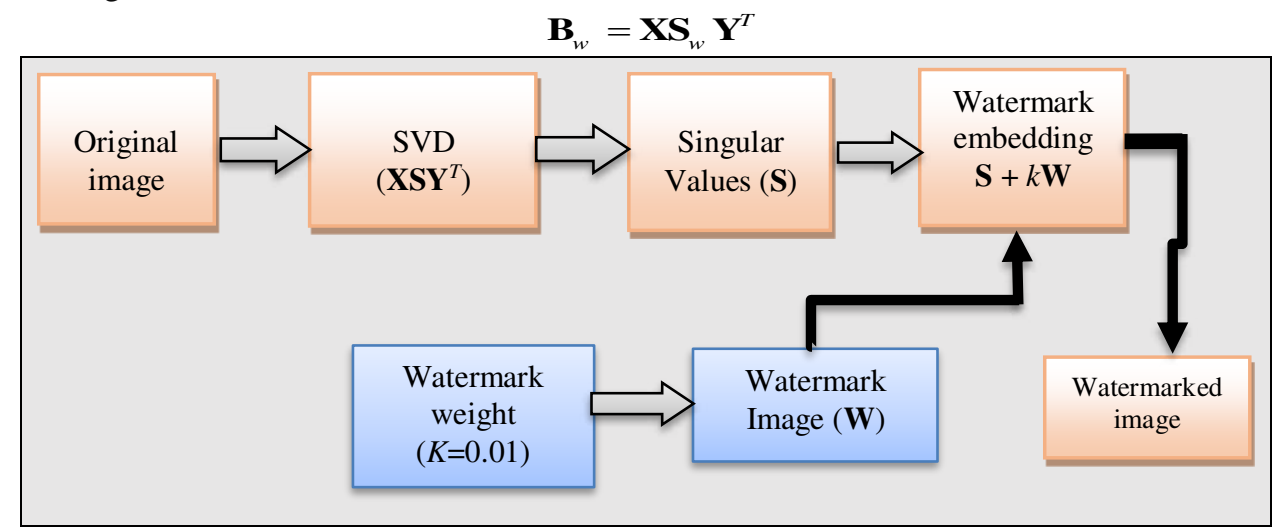

Fig. 3. The SVD watermark embedding process.

\subsubsection{Detection/Extraction Process:}

Having $\mathbf{X}_{w}, \mathbf{S}, \mathbf{Y}_{w}$ matrices and the possibly distorted image, the corrupted watermark is extracted from the watermarked image as follows:

1. The distorted watermarked image ( $\mathbf{B}_{w}^{*}$ matrix) is decomposed by SVD.

2. The matrix $\mathbf{D}^{*}$ is obtained.

$$
\mathbf{B}_{w}^{*}=\mathbf{X}^{*} \mathbf{S}_{w}^{*} \mathbf{Y}^{* T}
$$

3. Using the $\mathbf{D}^{*}$ and $\mathbf{S}$ matrices, the watermark is obtained.

$$
\mathbf{W}^{*}=\left(\mathbf{D}^{*}-\mathbf{S}\right) / K
$$

The corruption from attacks is referred to with *

\section{Proposed Hybrid Security and Error Control Framework}

In this section, the proposed hybrid watermarking and error control framework is introduced. The general framework of the proposed cornea and infrared video communication system is shown in Fig. 4. Firstly, the cornea and infrared frames are watermarked using the proposed embedding process to embed two watermarks in the host cornea and infrared frames. After that, the watermarked cornea and infrared frames are transmitted through a heavily corrupted wireless channel and received at the receiver side. Due to the channel noise effect on the transmitted watermarked cornea and infrared frames, they are affected by the wireless channel errors. So, we propose efficient post-processing error control algorithms at the receiver to conceal the errors in the received watermarked cornea and infrared frames, efficiently. The proposed error control algorithms have the advantage of neither needing modifications at the transmitter side nor increasing the transmission bit rate. After performing the error recovery process of the received corrupted watermarked cornea and infrared frames, the proposed extraction process is implemented on the resulting concealed watermarked cornea and infrared frames to extract the watermarks. The suggested embedding and extraction processes, the proposed post-processing error control schemes at the receiver side for the corrupted watermarked frames, and the proposed BKF enhancement algorithm will be discussed and explained in detail in the next sections. 


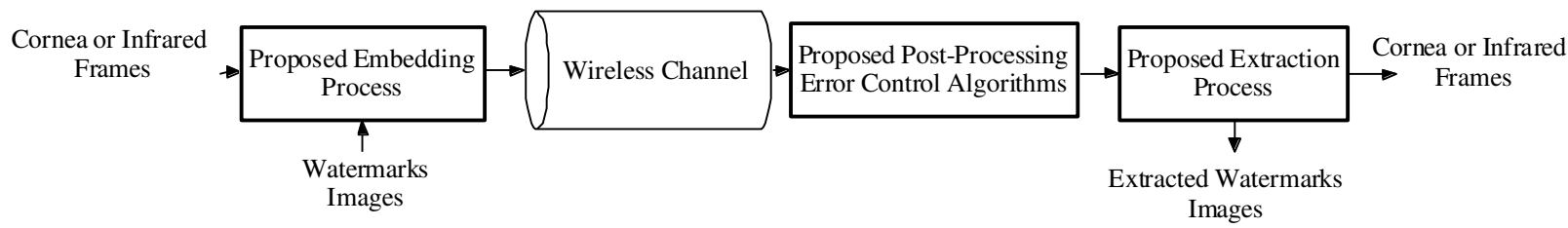

Fig. 4. General framework of the proposed hybrid security and error control framework for wireless cornea and infrared video communication.

\subsection{Proposed Hybrid Watermarking Algorithm (B-SVD/SVD)}

The proposed hybrid watermarking algorithm is based on the SVD and the BSVD. It satisfies the following watermarking requirements:

1. Improving the level of security of the algorithm.

2. Maintaining the imperceptibility, where the quality of the original image is not affected when increasing the capacity of information embedding.

3. Enhancing the watermark detectability.

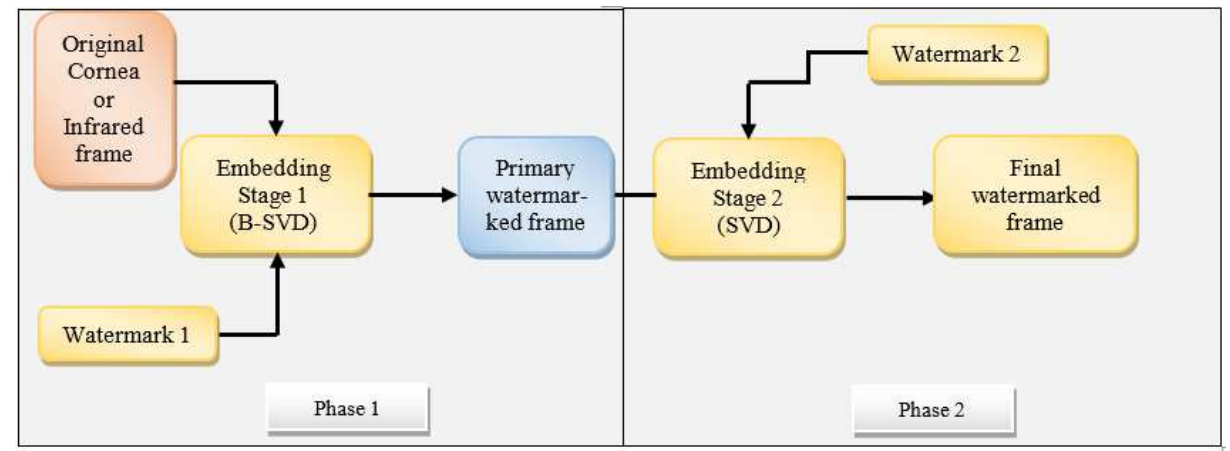

Fig. 5. General block diagram of the proposed embedding process.

The basic idea of the proposed algorithm is embedding two watermarks in the original cornea or infrared frames by using a hybrid B-SVD and SVD structure to produce the watermarked cornea and infrared frames. Briefly, the proposed hybrid watermarking algorithm consists of embedding and extraction processes as shown in the general block diagrams of Figs. 5 and 6. As shown in Fig. 5, to get the final watermarked frames, the embedding process consists of two stages. In the first stage of the embedding process, watermark 1 is embedded into the original cornea or infrared frame by using the B-SVD watermarking algorithm. Then, watermark 2 is embedded into the primarily watermarked frame by using the SVD watermarking algorithm. Figure 6 shows the extraction process, which consists of two stages. Firstly, watermark 2 is detected and extracted from the final watermarked frame. After that, watermark 1 is extracted in the second stage.

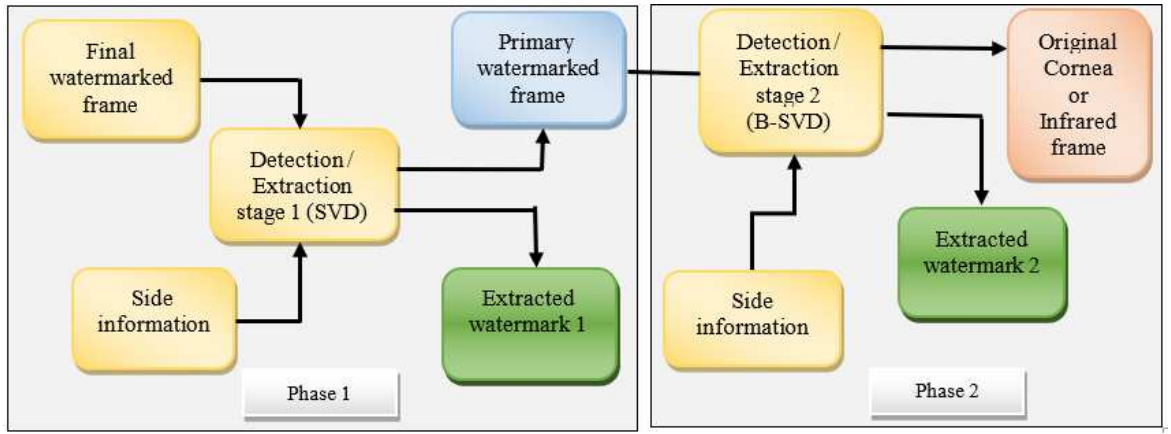

Fig. 6. The general block diagram of the proposed extraction process. 
The detailed steps of the proposed hybrid embedding/extraction processes are discussed in detail in the following sub-sections and shown in Figs. 7 and 8.

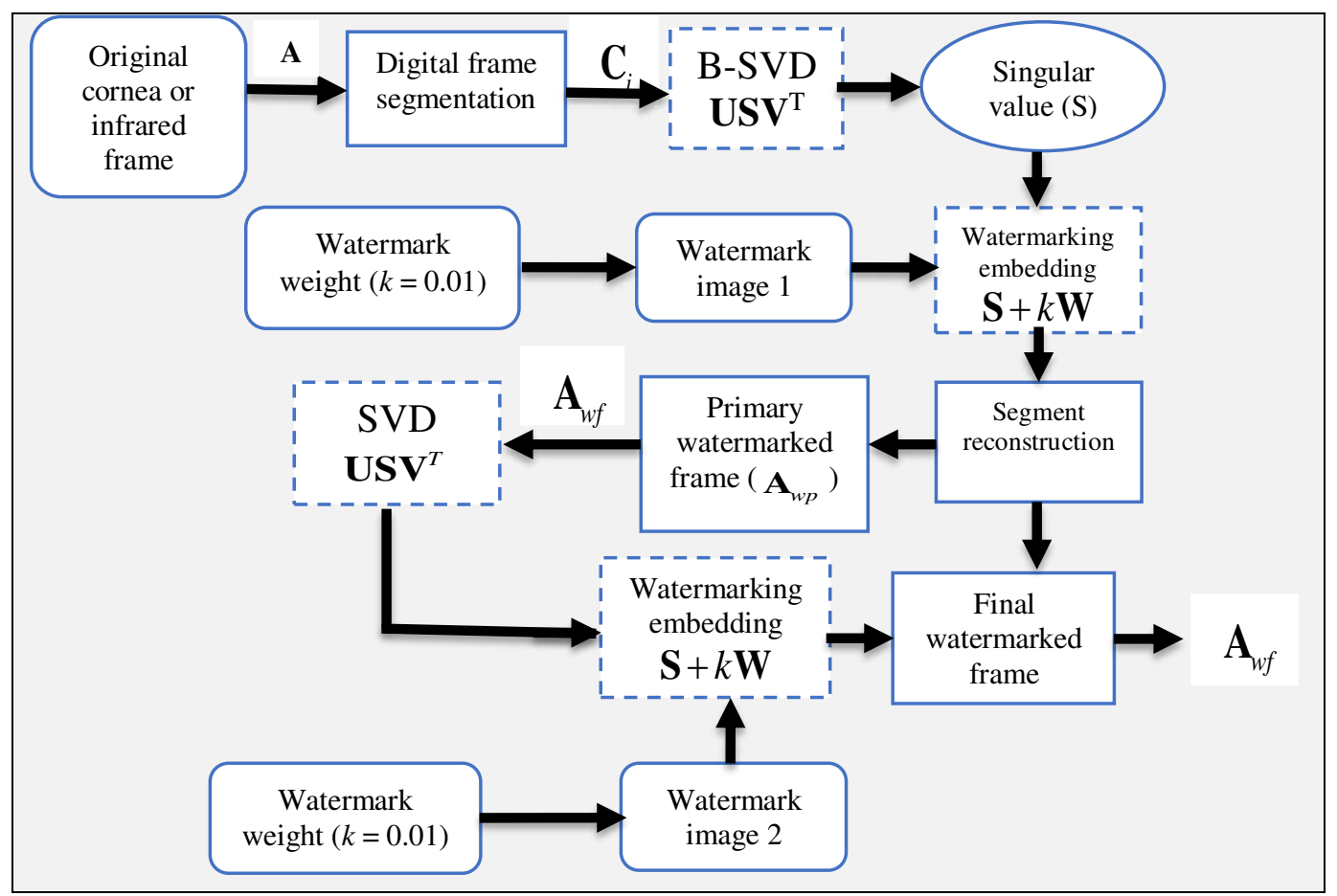

Fig. 7. The detailed steps of the proposed embedding process.

\subsubsection{Proposed Embedding Process:}

In the proposed embedding process, we apply the B-SVD watermarking and the SVD watermarking algorithms, respectively, as shown in Fig. 8 with the following steps:

1. The original cornea or infrared frame ( $\mathbf{A}$ matrix) is divided into non-overlapping blocks with the same size, and then the SVD is applied on each block $\left(\mathbf{C}_{i}\right.$ matrix) to obtain the SVs ( $\mathbf{S}_{i}$ matrix) of each block, where $i=1,2,3 . \ldots, N$ and $N$ is the number of blocks.

$$
\mathbf{C}_{i}=\mathbf{X}_{i} \mathbf{S}_{i} \mathbf{Y}_{i}^{T}
$$

2. The watermark image ( $\mathbf{W}$ matrix) is added to the $\mathbf{S}$ matrix of each block.

$$
\mathbf{D}_{i}=\mathbf{S}_{i}+K \mathbf{W}
$$

3. The SVs of each $\mathbf{S}_{w i}$ matrix are obtained by applying the SVD on each $\mathbf{D}_{i}$ matrix.

$$
\mathbf{D}_{i}=\mathbf{X}_{w i} \mathbf{S}_{w i} \mathbf{Y}_{w i}^{T}
$$

4. The watermarked blocks in the spatial domain are built using the $\mathrm{SV}$ s of each $\mathbf{D}_{i}$ matrix $\left(\mathbf{S}_{w i}\right.$ matrix).

$$
\mathbf{C}_{w i}=\mathbf{X}_{i} \mathbf{S}_{w i} \mathbf{Y}_{i}^{T}
$$

5. The watermarked frame in the time domain ( $\mathbf{A}_{w p}$ matrix) is built by combining the watermarked blocks back into one matrix.

From the previous steps of the first stage of the proposed algorithm, we get the primarily watermarked frame which is the input to the second stage having the following steps:

6. The SVD is applied on the primarily watermarked frame.

$$
\mathbf{A}_{w p}=\mathbf{X ~ \mathbf { S } _ { w p }} \mathbf{Y}^{T}
$$


7. To obtain the matrix $\mathbf{D}$, the watermark ( $\mathbf{W}$ matrix) is added to the SVs of the primarily watermarked matrix, and then the SVD is applied.

$$
\begin{aligned}
& \mathbf{D}=\mathbf{S}_{w p}+K \mathbf{W} \\
& \mathbf{D}=\mathbf{X}_{w} \mathbf{S}_{w p} \mathbf{Y}^{T}
\end{aligned}
$$

8. Using the modified matrix ( $\mathbf{S}_{w p}$ matrix), the final watermarked frame ( $\mathbf{A}_{w f}$ matrix) is obtained.

$$
\mathbf{A}_{w f}=\mathbf{X S}_{w p} \mathbf{Y}^{T}
$$

\subsubsection{Proposed Extraction Process:}

Figure 8 shows the extraction/detection process through the application of the SVD and B-SVD extraction algorithms, respectively. The proposed extraction process consists of the following steps:

1. The SVD is applied on the received final watermarked frame ( $\mathbf{A}_{w f}^{*}$ matrix).

$$
\mathbf{D}^{*}=\mathbf{X}_{w f} \mathbf{S}_{w f}^{*} \mathbf{Y}_{w f}^{T}
$$

2. The matrix containing the watermark is estimated.

$$
\mathbf{D}^{*}=\mathbf{X}_{w f} \mathbf{S}_{w f}^{*} \mathbf{Y}_{w f}^{T}
$$

3. The matrices $\mathbf{D}^{*}$ and $\mathbf{S}_{w f}$ are utilized to extract watermark 1, which may be corrupted.

$$
\mathbf{W} 1^{*}=\left(\mathbf{D}^{*}-\mathbf{S}_{w f}\right) / K
$$

To get the watermark 2, by knowing $\mathbf{X}_{w i}, \mathbf{Y}_{w i}, \mathbf{S}_{i}$ matrices and the possibly corrupted primarily watermarked frame $\mathbf{A}_{w p}^{*}$, we apply the steps below:

4. The corrupted primarily watermarked frame $\left(\mathbf{A}_{w p}^{*}\right)$ is divided into blocks having the same size as that utilized in the embedding process.

5. The SVs of each one ( $\mathbf{S}_{w p i}^{*}$ matrix) are obtained by applying SVD on each corrupted watermarked block ( $\mathbf{C}_{w p i}^{*}$ matrix).

$$
\mathbf{C}_{w p i}^{*}=\mathbf{X}_{i}^{*} \mathbf{S}_{w p i}^{*} \mathbf{Y}_{i}^{* T}
$$

6. The $\mathbf{X}_{w i}, \mathbf{Y}_{w i}$ and $\mathbf{S}_{w i}$ matrices are used to obtain the $\mathbf{C}_{w p i}^{*}$ matrices that contain watermark 2.

$$
\mathbf{D}_{i}^{*}=\mathbf{X}_{w p i} \mathbf{S}_{w p i}^{*} \mathbf{Y}_{w p i}^{T}
$$

7. From the $\mathbf{D}_{i}^{*}$ matrices, the corrupted watermark $2\left(\mathbf{W} 2_{i}^{*}\right.$ matrix $)$ is extracted.

$$
\left(\mathbf{D}_{i}^{*}-\mathbf{S}_{w p i}\right) / K=\mathbf{W} 2_{i}^{*}
$$




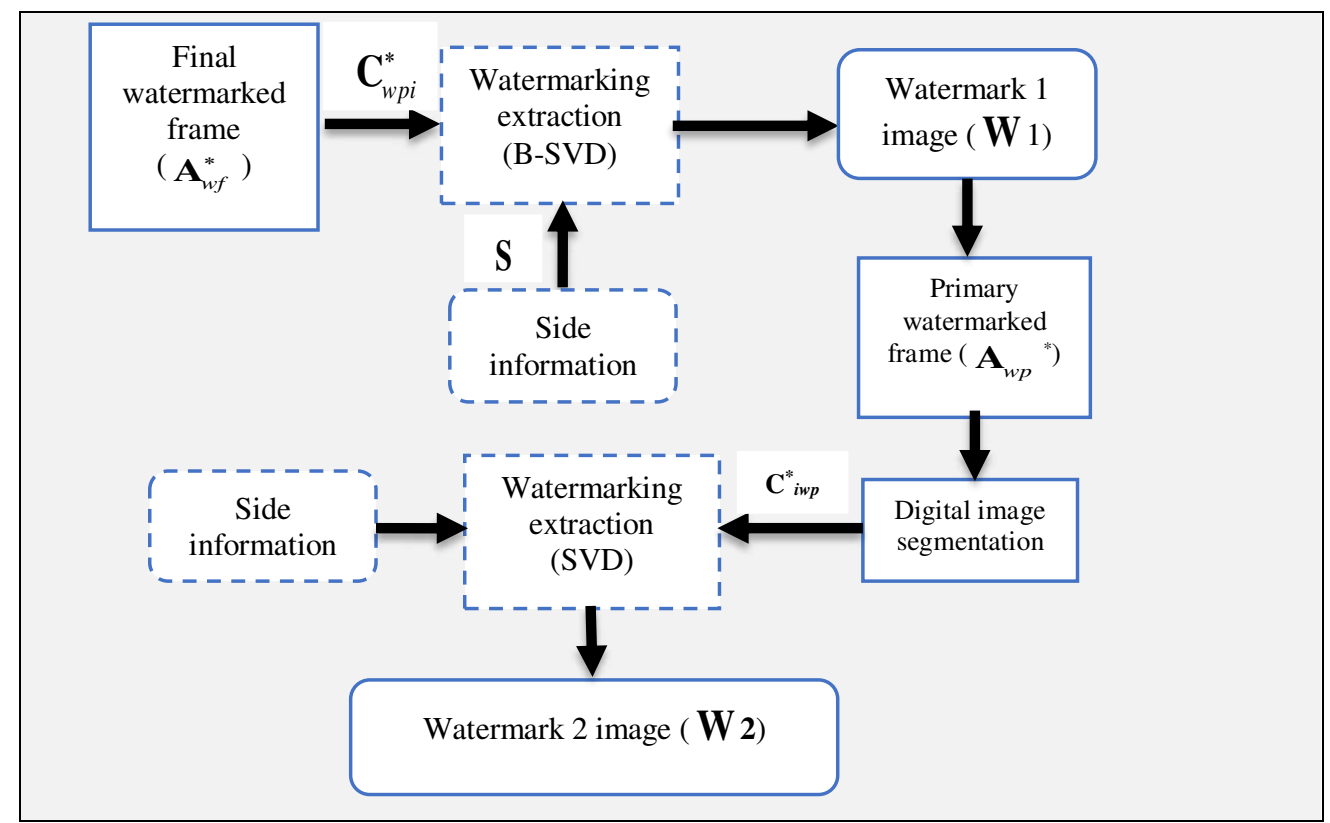

Fig. 8. The detail steps of the proposed watermark extraction process.

\subsection{Proposed Spatial and Temporal Post-processing Error Control Algorithms}

The temporal Outer Block Boundary Matching Algorithm (OBBMA) and the spatial Weight Pixel Averaging Interpolation (WPAI) techniques [31-35] were proposed for 2D video concealment. However, they fail and cannot work efficiently in the scenario of severely corrupted channels, and therefore they result in deterioration of the communication performance. Thus, we present an enhanced approach of temporal PMCA and spatial CSOIA procedures that proved efficiency in previous works [4] for efficient three-dimensional video transmission. So, in this work, we exploit both of them in the proposed framework for concealing the cornea and infrared frames for achieving an adequate error control performance.

Figure 9 shows the proposed hybrid framework of the spatial and temporal error control for the cornea and infrared frames. As shown, the spatial CSOIA is utilized for estimating the DVs of the corrupted MBs based on their neighboring and surrounding pixels. Also, the temporal PMCA is applied to determine the MVs of the lost MBs based on their neighboring and surrounding reference frames. After that, the Sum of Absolute Difference (SAD) is utilized to collect the primary concealment candidate MVs and DVs. Then, suitable coefficient magnitudes are marked for the collected MBs of the most optimum candidate DVs and MVs (MBopt. (DVs) and MBopt. (MVs)). Finally, the erroneous MBs are exchanged with the estimated optimal weighted DV and MV values of the candidate MBs. For more theoretical descriptions and mathematical details about the CSOIA and PMCA techniques, see [4]. Therefore, we use CSOIA and PMCA techniques in the current proposed work due to their advantages in mitigating the channel errors.

\subsection{Proposed BKF Enhancement Algorithm}

The proposed BKF enhancement algorithm is introduced in this section. It proved its efficiency in the previous work $[9,11]$ for efficient three-dimensional video transmission. In this work, it is exploited for smoothing the optimum formerly-determined DVs and MVs by the proposed spatial and temporal post-processing error control algorithms. It is used for compensating for the remnant 
measurement corruption among the chosen candidate DVs and MVs. Thus, the BKF is used as an amelioration stage for enhancement of the cornea and infrared frame visual quality.

Therefore, the stages of the proposed BKF algorithm [9] are iterated until the whole erroneous frames are recovered. Consequently, if the initially-determined DVs and MVs are not sufficient for an optimum error control process because of limited available surrounding pixel information and severe errors. Therefore, they are then enhanced by the predicted DVs and MVs, which are estimated through the utilization of the suggested BKF technique to achieve better cornea and infrared video transmission performance. More theoretical descriptions and mathematical details about the BKF algorithm can be found in our previous work in $[9,11]$. Therefore, we exploit it in the current proposed work due to its benefits and impact in lessening the communication errors.

\section{Simulation Results}

In order to appraise the efficiency of the suggested hybrid algorithms, we have carried out different simulation tests on cornea and infrared frames with and without attacks. For each video, the encoded cornea and infrared frames are obtained, and after that, they are watermarked using the proposed embedding process. After that, they are transmitted over a lossy wireless transmission channel, which is simulated using the Gilbert channel model [34]. Then, the received watermarked and corrupted cornea and infrared frames are subsequently decoded and concealed by the suggested post-processing error control techniques at the receiver side. After that, the proposed extraction process is employed on the concealed frames to extract the possibly corrupted watermarks. To assess the sufficiency of the suggested error control schemes, the PSNR, the Feature Similarity (FSIM) index, and the Structural Similarity (SSIM) index [35] are used as objective metrics to determine the quality of the reconstructed and concealed cornea and infrared frames. Further details about their definitions and equations can be found in [36].

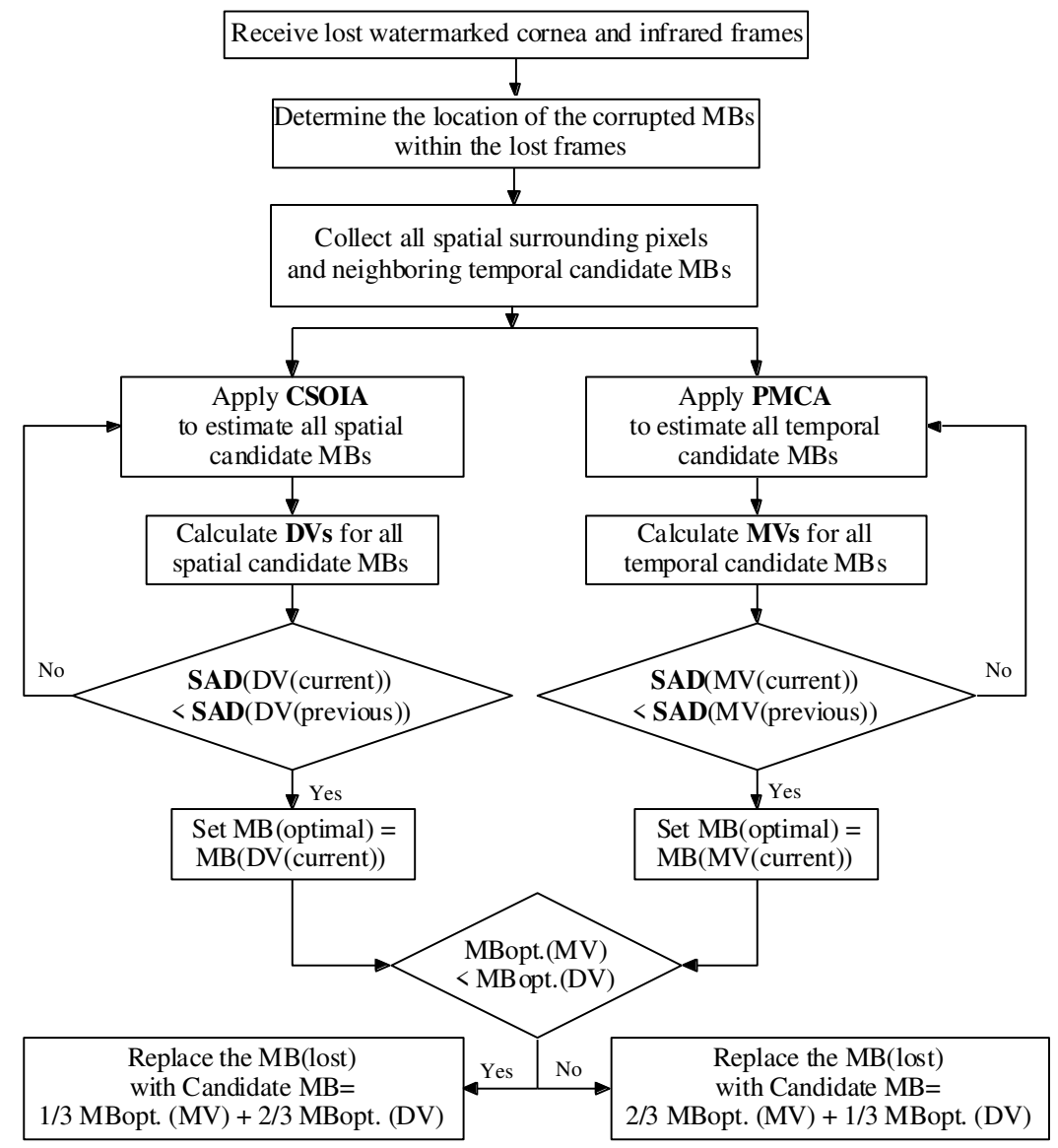


Fig. 9. Proposed framework of post-processing error control schemes.

To assess the efficiency of the proposed embedding/extraction processes, the visual results ensure watermark invisibility and no degradation in the watermarked frame quality compared to the original frames. Also, the PSNRs of the watermarked frames and the correlation coefficients $\left(C_{r}\right)$ of the extracted possibly corrupted watermarks with the original ones are estimated. The large PSNR value is considered as an imperceptibility quality metric. It should be infinite, but this is not possible for watermarked frames. The mathematical representation of the PSNR is given in (24), where $\mathbf{A}(x, y)$ is the original frame and $\mathbf{A}_{w}(x, y)$ is the watermarked frame. The $C_{r}$ value is considered as a robustness quality metric that measures the difference between the extracted and the original watermarks. The closer the $C_{r}$ value to one, the larger the robustness is $[37,38]$. The mathematical representation of the $C_{r}$ is given in (25), where $\mathbf{W}$ and $\mathbf{W}^{\prime}$ are the original and extracted watermarks, respectively. We have performed more simulation experiments on the tested cornea and infrared frames to carefully test the proposed hybrid algorithms, and thence we present the average values of the objective simulation results.

$$
\begin{gathered}
P S N R=10 \log _{10}\left(\frac{255^{2}}{\frac{1}{N^{2}} \sum_{x, y}\left(\mathbf{A}_{w}(x, y)-\mathbf{A}(x, y)\right)^{2}}\right) \\
C r\left(\mathbf{W}, \mathbf{W}^{\prime}\right)=\frac{\sum_{y} \mathbf{W}(y) \mathbf{W}^{\prime}(y)}{\sqrt{\sum_{y} \mathbf{W}^{2}(y) \sum_{y} \mathbf{W}^{\prime 2}(y)}}
\end{gathered}
$$

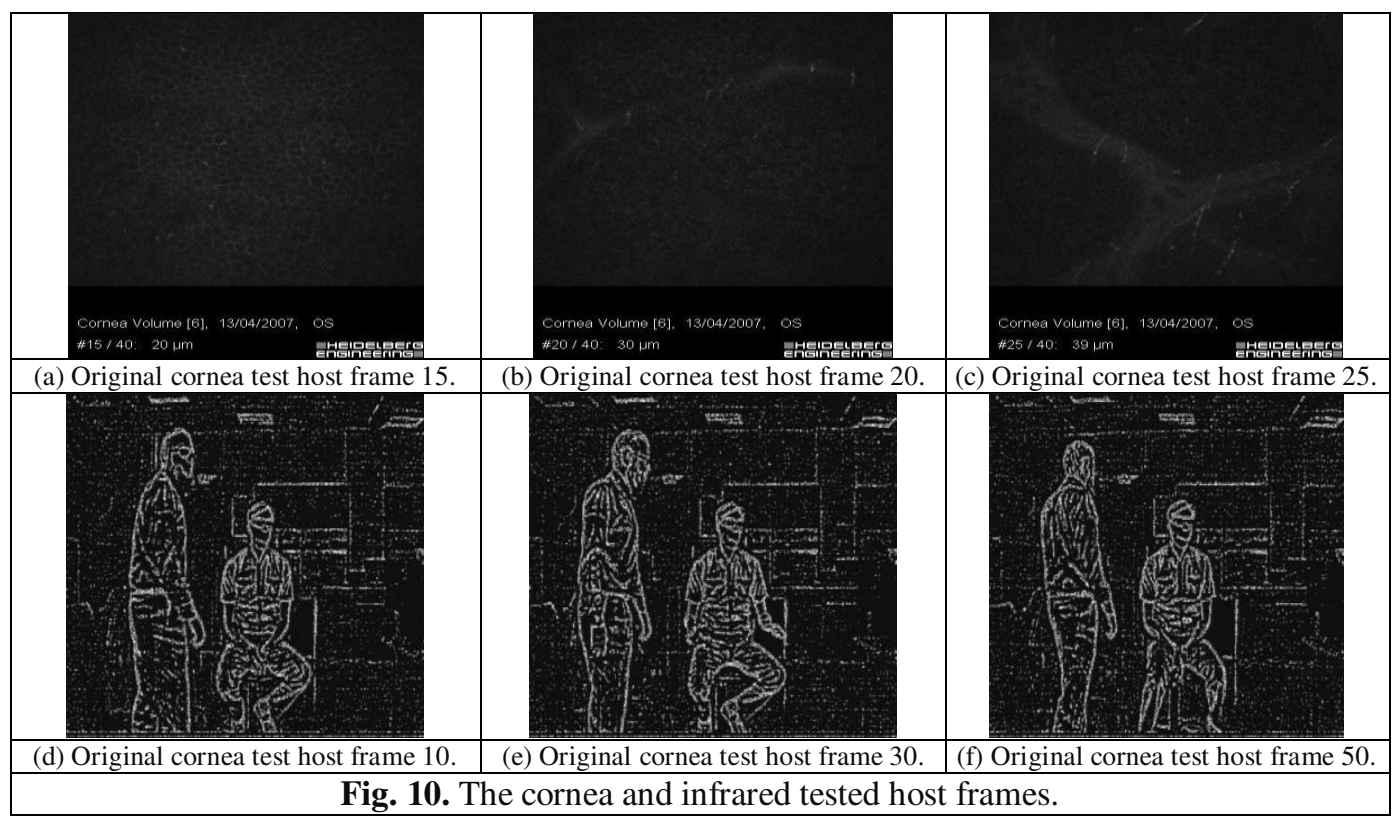

In our simulation experiments, we apply different types of multimedia attacks on the watermarked frames, and then extract the watermark to test the robustness of the proposed watermarking algorithm. We have run two experiments for the tested cornea and infrared videos. The first experiment on the cornea video was implemented on frames 15,20 , and 25 . The other experiment on the infrared video was implemented on frames 10, 30, and 50, as shown in Fig. 10. Figure 11 presents the utilized watermark images. 


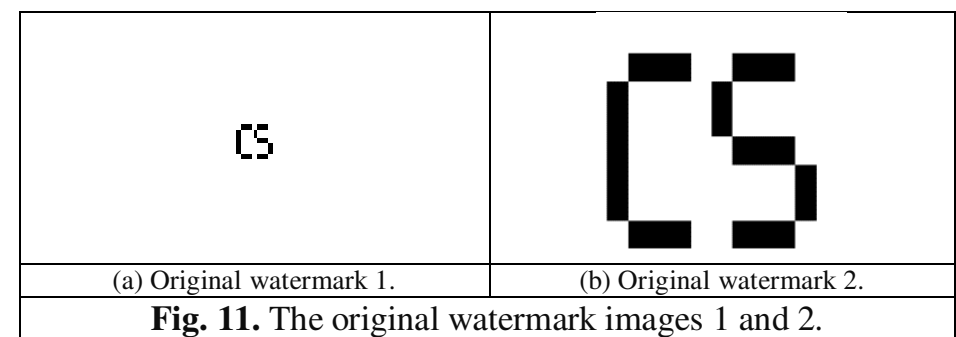

To clarify the efficiency of the proposed hybrid watermarking algorithm in protecting and securing the transmitted cornea and infrared frames in the presence of attacks, we have compared its performance, robustness, and imperceptibility with those of the state-of-the-art watermarking schemes such as SVD and DWT + SVD [37-40]. In the introduced simulation results, the B-SVD + SVD refers to the proposed hybrid watermarking algorithm.

In Tables 1, 2, 3, and 4, we compare the objective average PSNR values of the watermarked frames and the average $C_{r}$ values of the extracted watermark images of the cornea and infrared frames for the proposed hybrid watermarking algorithm and the stateof-the-art watermarking algorithms with and without employing different types of attacks. From the presented tables, it is noticed from the simulation results of the proposed watermarking algorithm compared to the state-of-the-art watermarking algorithms [3740] in the presence of different attacks that the proposed hybrid watermarking algorithm gives not only the highest PSNR values between the original and watermarked frames, but also the best correlation between the original and extracted watermarks. Therefore, it is clear from the presented results that there is a higher similarity between the original and watermarked frames. The proposed algorithm also improves the similarity between the original and extracted watermarks. Moreover, the proposed algorithm achieves high PSNR and $C_{r}$ values for all tested cornea and infrared frames.

Table 1 Objective average PSNR values of the watermarked frames for the cornea frames with and without attacks.

\begin{tabular}{|c|c|c|c|c|c|}
\hline \multicolumn{7}{|c|}{ Average PSNR for watermarking algorithms } \\
\hline $\begin{array}{c}\text { Attack } \\
\text { type }\end{array}$ & $\begin{array}{c}\text { SVD only with one } \\
\text { watermark [37] }\end{array}$ & $\begin{array}{c}\text { DWT+SVD with } \\
\text { one watermark } \\
{[\mathbf{3 8}]}\end{array}$ & $\begin{array}{c}\text { SVD only with } \\
\text { fused } \\
\text { watermark [40] }\end{array}$ & $\begin{array}{c}\text { DWT+SVD with } \\
\text { fused watermark } \\
{[\mathbf{3 9}]}\end{array}$ & $\begin{array}{c}\text { B-SVD+SVD } \\
\text { (Proposed) }\end{array}$ \\
\hline No attack & 60.5775 & 61.1391 & 63.8077 & 64.3155 & 69.3076 \\
\hline Gaussian noise & 20.3733 & 20.4082 & 20.404 & 20.4017 & 22.6902 \\
\hline LPF & 20.6968 & 20.6968 & 20.6921 & 20.6928 & 22.6902 \\
\hline Crop & 7.8124 & 7.8124 & 7.8116 & 7.8166 & 20.7841 \\
\hline
\end{tabular}

Table 2 Objective average $C r$ values of the extracted watermark images for the cornea frames with and without attacks.

\begin{tabular}{|c|c|c|c|c|c|}
\hline \multicolumn{7}{|c|}{ Average Cr for watermarking algorithms } \\
\hline $\begin{array}{c}\text { Attack } \\
\text { type }\end{array}$ & $\begin{array}{c}\text { SVD only with one } \\
\text { watermark [37] }\end{array}$ & $\begin{array}{c}\text { DWT+SVD with } \\
\text { one watermark } \\
{[\mathbf{3 8}]}\end{array}$ & $\begin{array}{c}\text { SVD only with } \\
\text { fused } \\
\text { watermark [40] }\end{array}$ & $\begin{array}{c}\text { DWT+SVD with } \\
\text { fused watermark } \\
{[\mathbf{3 9}]}\end{array}$ & $\begin{array}{c}\text { B-SVD+SVD } \\
\text { (Proposed) }\end{array}$ \\
\hline No attack & 0.9926 & 0.9968 & 0.8508 & 1 & 0.9989 \\
\hline Gaussian noise & 0.4716 & 0.5842 & 0.1373 & 0.5124 & 0.5963 \\
\hline LPF & 0.4366 & 0.6927 & 0.067 & 0.136 & 0.7751 \\
\hline Crop & 0.9918 & 0.8997 & 0.0106 & 0.2529 & 0.9989 \\
\hline
\end{tabular}

Table 3 Objective average PSNR values of the watermarked frames for the infrared frames with and without attacks.

\begin{tabular}{|c|c|c|c|c|c|}
\hline \multicolumn{7}{|c|}{ Average PSNR for watermarking algorithms } \\
\hline $\begin{array}{c}\text { Attack } \\
\text { type }\end{array}$ & $\begin{array}{c}\text { SVD only with one } \\
\text { watermark [37] }\end{array}$ & $\begin{array}{c}\text { DWT+SVD with } \\
\text { one watermark } \\
{[\mathbf{3 8}]}\end{array}$ & $\begin{array}{c}\text { SVD only with } \\
\text { fused } \\
\text { watermark [40] }\end{array}$ & $\begin{array}{c}\text { DWT+SVD with } \\
\text { fused watermark } \\
{[\mathbf{3 9}]}\end{array}$ & $\begin{array}{c}\text { B-SVD+SVD } \\
\text { (Proposed) }\end{array}$ \\
\hline No attack & 60.5775 & 61.1391 & 63.8077 & 64.3155 & 52.2784 \\
\hline Gaussian noise & 20.3733 & 20.4082 & 20.404 & 20.4017 & 32.3079 \\
\hline
\end{tabular}




\begin{tabular}{|c|c|c|c|c|c|}
\hline LPF & 20.6968 & 20.6968 & 20.6921 & 20.6928 & 32.3079 \\
\hline Crop & 7.8124 & 7.8124 & 7.8116 & 7.8166 & 28.1868 \\
\hline
\end{tabular}

Table 4 Objective average $\mathrm{Cr}$ values of the extracted watermark images for the infrared frames with and without employing different attacks.

\begin{tabular}{|c|c|c|c|c|c|}
\hline \multicolumn{6}{|c|}{ Average Cr for watermarking algorithms } \\
\hline $\begin{array}{c}\text { Attack } \\
\text { type }\end{array}$ & $\begin{array}{c}\text { SVD only with one } \\
\text { watermark [37] }\end{array}$ & $\begin{array}{c}\text { DWT+SVD with } \\
\text { one watermark } \\
\text { [38] }\end{array}$ & $\begin{array}{c}\text { SVD only with } \\
\text { fused } \\
\text { watermark [40] }\end{array}$ & $\begin{array}{c}\text { DWT+SVD with } \\
\text { fused watermark } \\
\text { [39] }\end{array}$ & $\begin{array}{c}\text { B-SVD+SVD } \\
\text { (Proposed) }\end{array}$ \\
\hline No attack & 0.9926 & 0.8969 & 0.8508 & 1 & 0.9979 \\
\hline Gaussian noise & 0.4716 & 0.5743 & 0.1373 & 0.5124 & 0.5963 \\
\hline LPF & 0.4366 & 0.7472 & 0.067 & 0.136 & 0.7751 \\
\hline Crop & 0.9918 & 0.9246 & 0.0106 & 0.2529 & 0.9989 \\
\hline
\end{tabular}

To show the good performance of the proposed hybrid error control algorithms, we have run further comparative simulation experiments between the proposed hybrid error control algorithms and the traditional algorithms in [12-14, 32-35] for the cornea and infrared frames at PLR=40\%. The PSNR, FSIM, and SSIM numerical results are presented to validate the performance of the suggested algorithms compared to the traditional ones. Table 5 shows the average PSNR, FSIM, and SSIM of the proposed algorithms compared to the algorithms in $[12-14,32-35]$ at PLR=40\%. It can be seen that the proposed algorithms outperform the state-of-the-art ones. So, it is observed that with the proposed error control algorithms, the recovery quality can be further enhanced by exploiting the hybrid structure of CSOIA, PMCA, and BKF algorithms. Therefore, the significance of exploiting the proposed error control algorithms for reconstructing the corrupted cornea and infrared frames sufficiently is clear in the scenario of severelycorrupting channels. From all presented simulation results on the tested cornea and infrared frames, we perceive that the suggested post-processing error control algorithms always achieve superior PSNR, FSIM, and SSIM values.

Table 5 Average PSNR (dB), FSIM, and SSIM objective comparison between the proposed error control algorithms and the stateof-the-art ones on the tested cornea and infrared frames.

\begin{tabular}{|c|c|c|c|c|c|c|c|c|}
\hline \multirow{2}{*}{ Frames } & \multicolumn{8}{|c|}{ PSNR (dB)/FSIM/SSIM } \\
\hline & [12] & [13] & [14] & [32] & [33] & [34] & [35] & Proposed \\
\hline Cornea & $\begin{array}{c}32.53 / 0.9235 / \\
0.9102\end{array}$ & $\begin{array}{c}35.67 / 0.9572 / \\
0.9352\end{array}$ & $\begin{array}{c}32.68 / 0.9420 / \\
0.91867\end{array}$ & $\begin{array}{c}37.67 / 0.9442 / \\
0.9358\end{array}$ & $\begin{array}{c}34.43 / 0.9573 / \\
0.9425\end{array}$ & $\begin{array}{c}35.85 / 0.9556 / \\
0.9472\end{array}$ & $\begin{array}{c}36.38 / 0.9461 / \\
0.9214\end{array}$ & $\begin{array}{c}40.79 / 0.9658 / \\
0.9531\end{array}$ \\
\hline Infrared & $\begin{array}{c}37.58 / 0.9235 / \\
0.9196\end{array}$ & $\begin{array}{c}39.82 / 0.9207 / \\
0.9192\end{array}$ & $\begin{array}{c}34.18 / 0.9435 / \\
0.9407\end{array}$ & $\begin{array}{c}38.37 / 0.9234 / \\
0.9372\end{array}$ & $\begin{array}{c}37.47 / 0.9273 / \\
0.9402\end{array}$ & $\begin{array}{c}39.62 / 0.9386 / \\
0.9270\end{array}$ & $\begin{array}{c}38.95 / 0.9221 / \\
0.9327\end{array}$ & $\begin{array}{c}42.86 / 0.9496 / \\
0.9428\end{array}$ \\
\hline
\end{tabular}

Also, it is important to reveal the effect of the utilization of the error control algorithms in the watermark detection process. To demonstrate the impact of employing the suggested error control algorithms in efficiently extracting the watermarks from the watermarked infrared and cornea frames, we perform different simulation tests to check the performance of watermark extraction with and without using the proposed hybrid error control algorithms for the cornea and infrared frames. Table 6 presents the average $C_{r}$ simulation results of the extracted watermarks from the watermarked cornea and infrared frames with and without exploiting the proposed hybrid error control algorithms in the presence of different types of attacks with a packet loss rate of $40 \%$. The significance of exploiting the proposed error control algorithms for a higher possibility of watermark extraction with higher correlation coefficient values from the corrupted cornea and infrared frames is clear in the scenario of attacks and severely-corrupting channels as in the presented state of PLR $=40 \%$.

Table 6 Objective average $C_{r}$ values of the extracted watermark images for the cornea and infrared frames with and without exploiting the proposed hybrid error control algorithms in the presence of different attacks at PLR=40\%. 


\begin{tabular}{|c|c|c|c|c|}
\hline \multicolumn{5}{|c|}{ Average Cr values } \\
\hline \multirow{2}{*}{$\begin{array}{c}\text { Attack } \\
\text { type }\end{array}$} & $\begin{array}{c}\text { Cornea frames } \\
\text { Watermark } \\
\text { extraction } \\
\text { without using } \\
\text { error control } \\
\text { algorithms }\end{array}$ & $\begin{array}{c}\text { Watermark } \\
\text { extraction } \\
\text { with using } \\
\text { error control } \\
\text { algorithms }\end{array}$ & $\begin{array}{c}\text { Watermark } \\
\text { extraction } \\
\text { without } \\
\text { using error } \\
\text { control } \\
\text { algorithms }\end{array}$ & $\begin{array}{c}\text { Watermark } \\
\text { extraction } \\
\text { with using } \\
\text { error } \\
\text { control } \\
\text { algorithms }\end{array}$ \\
\hline $\begin{array}{c}\text { No } \\
\text { attack }\end{array}$ & 0.7652 & 0.9989 & 0.7594 & 0.9979 \\
\hline $\begin{array}{c}\text { Gaussian } \\
\text { noise }\end{array}$ & 0.3658 & 0.5963 & 0.4205 & 0.5963 \\
\hline LPF & 0.5692 & 0.7751 & 0.6327 & 0.7751 \\
\hline Crop & 0.8035 & 0.9989 & 0.8348 & 0.9989 \\
\hline
\end{tabular}

\section{Conclusions}

This paper presented an efficient hybrid error control and watermarking algorithm for reliable cornea and infrared frame communication through wireless channels. The evaluation metrics for the proposed watermarking algorithm includes stability, reliability, and robustness. Experimental results revealed the superiority of the proposed hybrid watermarking algorithm in maintaining high robustness and fidelity in the presence of multimedia attacks compared to the existing watermarking algorithms. Moreover, the simulation results proved the importance of the suggested post-processing error control techniques on recovering the erroneous cornea and infrared frames for achieving significant PSNR, SSIM, and FSIM results. From all obtained results, we also ensured the importance of employing both proposed error control and security techniques for efficient transmission of medical data and infrared frames over wireless channels for secure and reliable telecommunication and IoT applications.

\section{Compliance with ethical standards}

Conflict of interest The authors declare that they have no conflict of interest.

\section{References}

1. H. Zeng, X. Wang, C. Cai, J. Chen and Y. Zhang, Fast multiview video coding using adaptive prediction structure and hierarchical mode decision, IEEE Trans. Circuits and Systems for Video Technology, 24(9) (2015) 1566-1578.

2. W. Xiang, P. Gao and Q. Peng, Robust multiview three-dimensional video communications based on distributed video coding, IEEE Systems Journal, PP(99) (2015) 1-11.

3. A. I. Purica, E. G. Mora, B. Pesquet-Popescu, M. Cagnazzo and B. Ionescu, Multiview plus depth video coding with temporal prediction view synthesis, IEEE Trans. Circuits and Systems for Video Technology, 26(2) (2016) 360-374.

4. El-Shafai, W., El-Rabaie, S., El-Halawany, M. M., \& Abd El-Samie, F. E. (2018). Performance evaluation of enhanced error correction algorithms for efficient wireless 3D video communication systems. International Journal of Communication Systems, 31(1), e3396.

5. A. De Abreu, P. Frossard and F. Pereira, Optimizing multiview video plus depth prediction structures for interactive multiview video streaming, IEEE Journal of Selected Topics in Signal Processing, 9(3) (2015) 487-500.

6. C. T. E. R. Hewage and M. G. Martini, Quality of experience for 3D video streaming, IEEE Communications Magazine, 51(5) (2013) 101-107.

7. Peled, A., Carny, O., Baratz, A., Arbel, O., \& Troyansky, L. (2009). U.S. Patent No. 7,627,897. Washington, DC: U.S. Patent and Tradem 
8. Battiato, S., Emmanuel, S., Ulges, A., \& Worring, M. (2012). Multimedia in forensics, security, and intelligence. IEEE MultiMedia, 19(1), 17-19.

9. El-Shafai, W., El-Rabaie, S., El-Halawany, M. M., \& El-Samie, F. E. A. (2019). Effective multi-stage error control algorithms for robust 3D video transmission over wireless networks. Wireless Networks, 25(4), 1619-1640.

10. Z. Liu, G. Cheung and Y. Ji, Optimizing distributed source coding for interactive multiview video streaming over lossy networks, IEEE Trans. on Circuits and Systems for Video Technology, 23(10) (2013) 1781-1794.

11. El-Shafai, W., El-Rabaie, S., El-Halawany, M. M., \& El-Samie, F. E. A. (2018). Encoder-independent decoder-dependent depth-assisted error concealment algorithm for wireless 3D video communication. Multimedia Tools and Applications, 77(11), 13145-13172.

12. S. Khattak, T. Maugey, R. Hamzaoui, S. Ahmad and P. Frossard, Temporal and inter-view consistent error concealment technique for multiview plus depth video, IEEE Trans. on Circuits and Systems for Video Technology, 26(5) (2016) 829-840.

13. Y. Zhou, W. Xiang and G. Wang, Frame loss concealment for multiview video transmission over wireless multimedia sensor networks, IEEE Sensors Journal, 15(3) (2015) 1892-1901.

14. P. J. Lee, K. T. Kuo and C. Y. Chi, An adaptive error concealment method based on fuzzy reasoning for multi-view video coding, Journal of Display Technology, 10(7) (2014) 560-567.

15. Gutub, A., Al-Juaid, N., \& Khan, E. (2019). Counting-based secret sharing technique for multimedia applications. Multimedia Tools and Applications, 78(5), 5591-5619.

16. Thakur, S., Singh, A. K., Ghrera, S. P., \& Elhoseny, M. (2019). Multi-layer security of medical data through watermarking and chaotic encryption for tele-health applications. Multimedia tools and Applications, 78(3), 3457-3470.

17. Zhang, J., Gu, Z., Jang, J., Wu, H., Stoecklin, M. P., Huang, H., \& Molloy, I. (2018, May). Protecting intellectual property of deep neural networks with watermarking. In Proceedings of the 2018 on Asia Conference on Computer and Communications Security (pp. 159-172). ACM.

18. Singh, A. K., Kumar, B., Singh, S. K., Ghrera, S. P., \& Mohan, A. (2018). Multiple watermarking technique for securing online social network contents using back propagation neural network. Future Generation Computer Systems, 86, $926-939$.

19. Zear, A., Singh, A. K., \& Kumar, P. (2018). Multiple watermarking for healthcare applications. Journal of Intelligent Systems, $27(1), 5-18$.

20. Wolff, L. B., Socolinsky, D. A., \& Eveland, C. K. (2009). U.S. Patent No. 7,620,265. Washington, DC: U.S. Patent and Trademark Office.

21. White, T. L., Lewis, P. N., Young, R. D., Kitazawa, K., Inatomi, T., Kinoshita, S., \& Meek, K. M. (2017). Elastic microfibril distribution in the cornea: differences between normal and keratoconic stroma. Experimental eye research, 159, 40-48.

22. Tan, I. J., Dobson, L. P., Bartnik, S., Muir, J., \& Turner, A. W. (2017). Real-time teleophthalmology versus face-to-face consultation: A systematic review. Journal of telemedicine and telecare, 23(7), 629-638.

23. Carruthers, J. B. (2003). Wireless infrared communications. Wiley Encyclopedia of Telecommunications.

24. Balasubramanian, M., Perkins, A. L., Beuerman, R. W., \& Iyengar, S. S. (2006, August). Fractal dimension based corneal fungal infection diagnosis. In Applications of Digital Image Processing XXIX (Vol. 6312, p. 631214). International Society for Optics and Photonics.

25. Fabijańska, A. (2017, September). Corneal endothelium image segmentation using feedforward neural network. In 2017 Federated Conference on Computer Science and Information Systems (FedCSIS) (pp. 629-637). IEEE.

26. Bucht, C., Söderberg, P., \& Manneberg, G. (2010, March). Fully automated corneal endothelial morphometry of images captured by clinical specular microscopy. In Ophthalmic Technologies XX (Vol. 7550, p. 75501E). International Society for Optics and Photonics.

27. J. Liu, Y. Zhang, X. Zheng and J. Song, A dynamic hybrid UXP/ARQ method for scalable video transmission, in IEEE 23rd Int. Symposium on Personal, Indoor and Mobile Radio Communications-(PIMRC) (IEEE, 2012), pp. 2566-2571. 
28. O. H. Salim, W. Xiang and J. Leis, An efficient unequal error protection scheme for 3-D video transmission, in 2013 IEEE Wireless Communications and Networking Conf. (WCNC) (IEEE, 2013), pp. 4077-4082.

29. Y. Huo, M. El-Hajjar and L. Hanzo, Inter-Layer FEC Aided Unequal Error Protection for Multilayer Video Transmission in Mobile TV, IEEE Trans. on Circuits and Systems for Video Technology, 23(9) (2013) 1622-1634.

30. Liu, R., \& Tan, T. (2002). An SVD-based watermarking scheme for protecting rightful ownership. IEEE transactions on multimedia, 4(1), 121-128.

31. Z. W. Gao and W. N. Lie, Video error concealment by using Kalman-filtering technique, in IEEE Int. Symposium on Circuits and Systems (IEEE, 2004), pp. II-69-72 Vol.2.

32. S. Cui, C. Huijuan and T. Kun, An effective error concealment scheme for heavily corrupted H.264/AVC videos based on Kalman filtering, Journal of Signal, Image and Video Processing, 8(8) (2012) 1533-1542.

33. M. C. Hwang, J. H. Kim, D. T. Duong and S. J. Ko, Hybrid temporal error concealment methods for block-based compressed video transmission, IEEE Trans. on Broadcasting, 54(2) (2008) 198-207.

34. W. El Shafai, B. Hrušovský, M. El-Khamy, M. El-Sharkawy, Joint space-time-view error concealment algorithms for 3D multi-view video, in: 18th Int. Conference on Image Processing (ICIP) (IEEE, 2011), pp 2201-2204.

35. W.N. Lie, C.M. Lee, C.H. Yeh, Z.W. Gao, Motion vector recovery for video error concealment by using iterative dynamicprogramming optimization, IEEE Transactions on Multimedia, 16(1) (2014) 216-227.

36. L. Zhang, L. Zhang, X. Mou, and D. Zhang, FSIM: A feature similarity index for image quality assessment, IEEE Trans. on Image Processing, 20(8) (2011) 2378-2386.

37. Dogan, S., Tuncer, T., Avci, E., \& Gulten, A. (2011). A robust color image watermarking with Singular Value Decomposition method. Advances in Engineering Software, 42(6), 336-346.

38. Bhatnagar, G., \& Raman, B. (2009). A new robust reference watermarking scheme based on DWT-SVD. Computer Standards \& Interfaces, 31(5), 1002-1013.

39. Hemdan, E. E. D., El-Fishawy, N., Attiya, G., \& El-samie, F. A. (2013, April). C11. Hybrid Digital Image Watermarking Technique for Data Hiding. In 2013 30th National Radio Science Conference (NRSC) (pp. 220-227). IEEE.

40. Singh, A. K., Dave, M., \& Mohan, A. (2016). Hybrid technique for robust and imperceptible multiple watermarking using medical images. Multimedia Tools and Applications, 75(14), 8381-8401. 


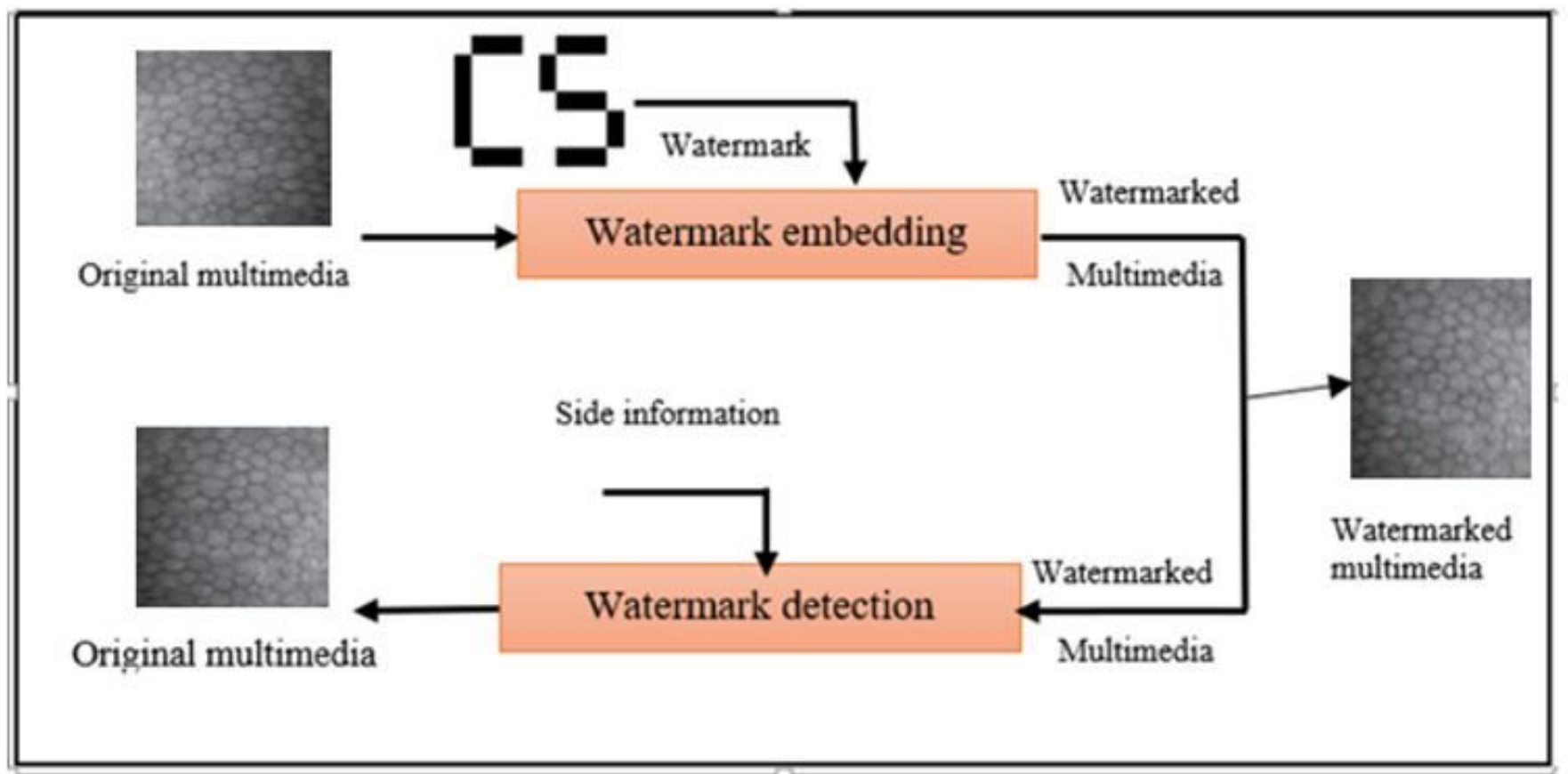

\section{Figure 1}

Watermarking system for multimedia content.

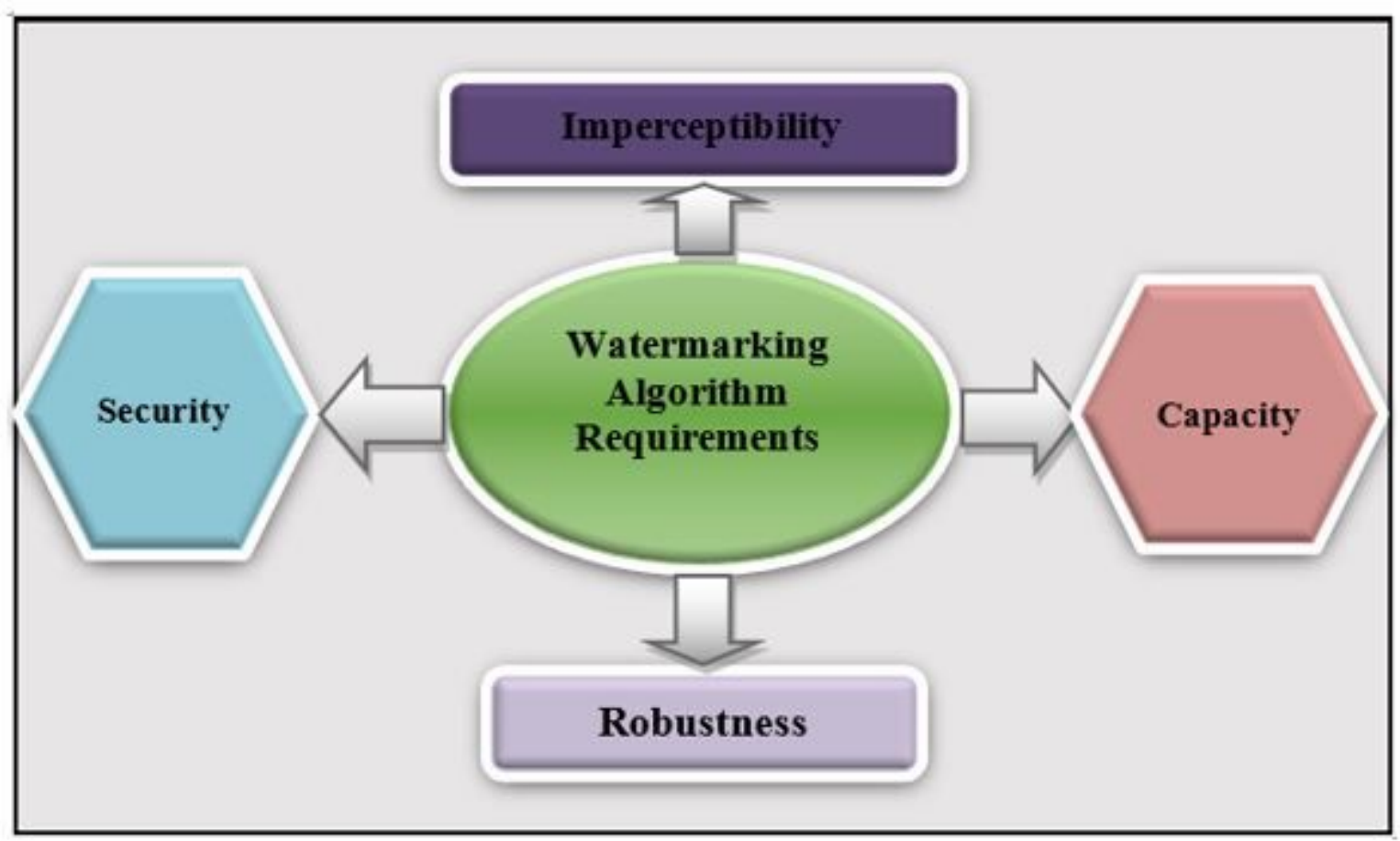

Figure 2

Multimedia watermarking requirements. 


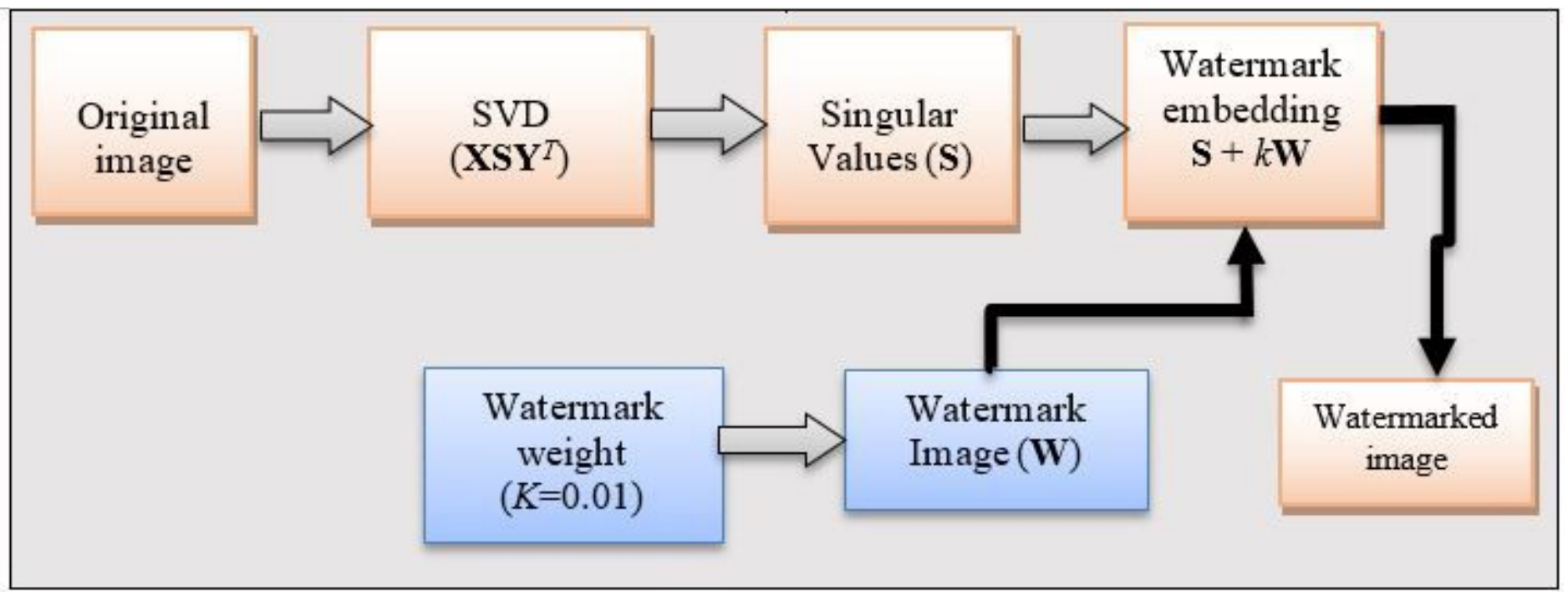

\section{Figure 3}

The SVD watermark embedding process.

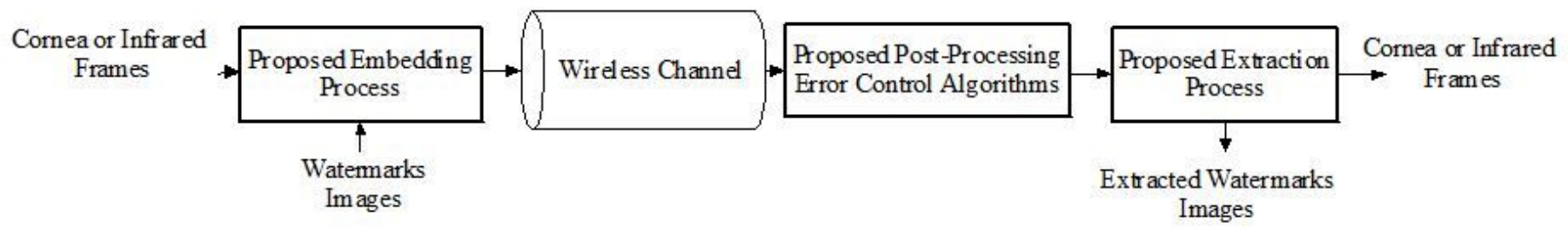

Figure 4

General framework of the proposed hybrid security and error control framework for wireless cornea and infrared video communication.



\section{Figure 5}

General block diagram of the proposed embedding process. 


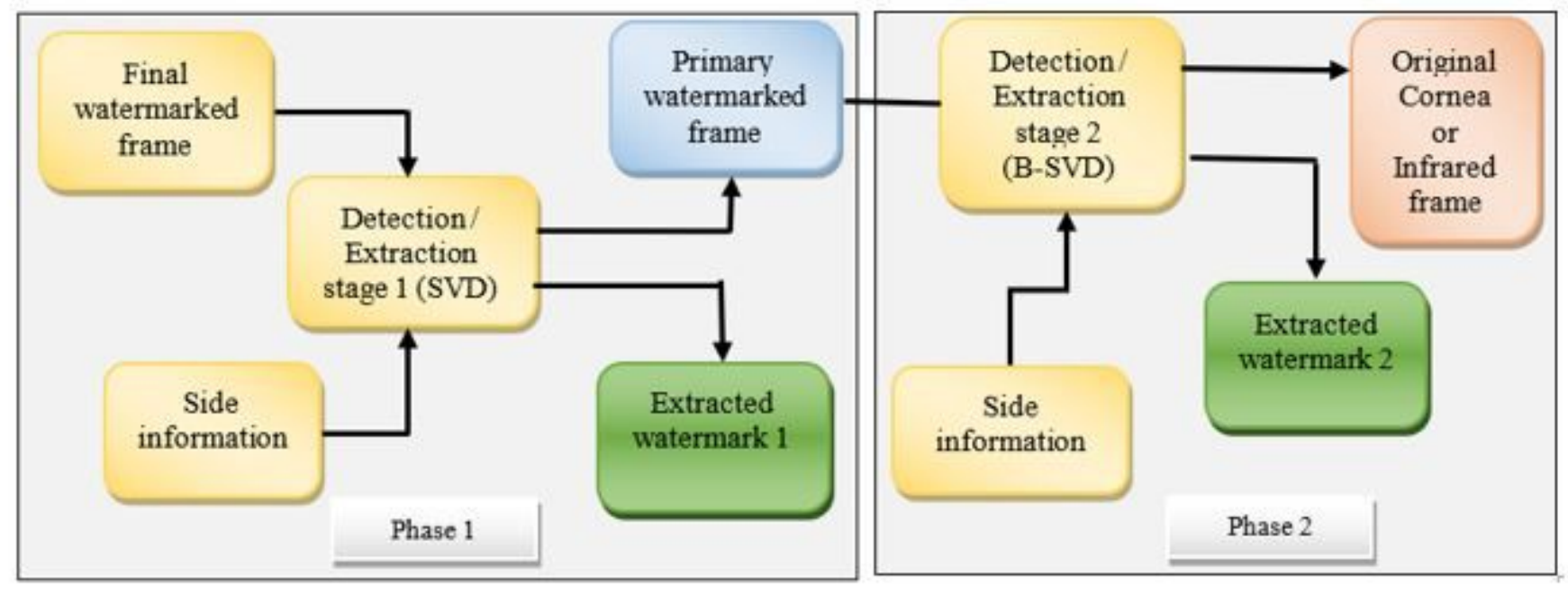

Figure 6

The general block diagram of the proposed extraction process.

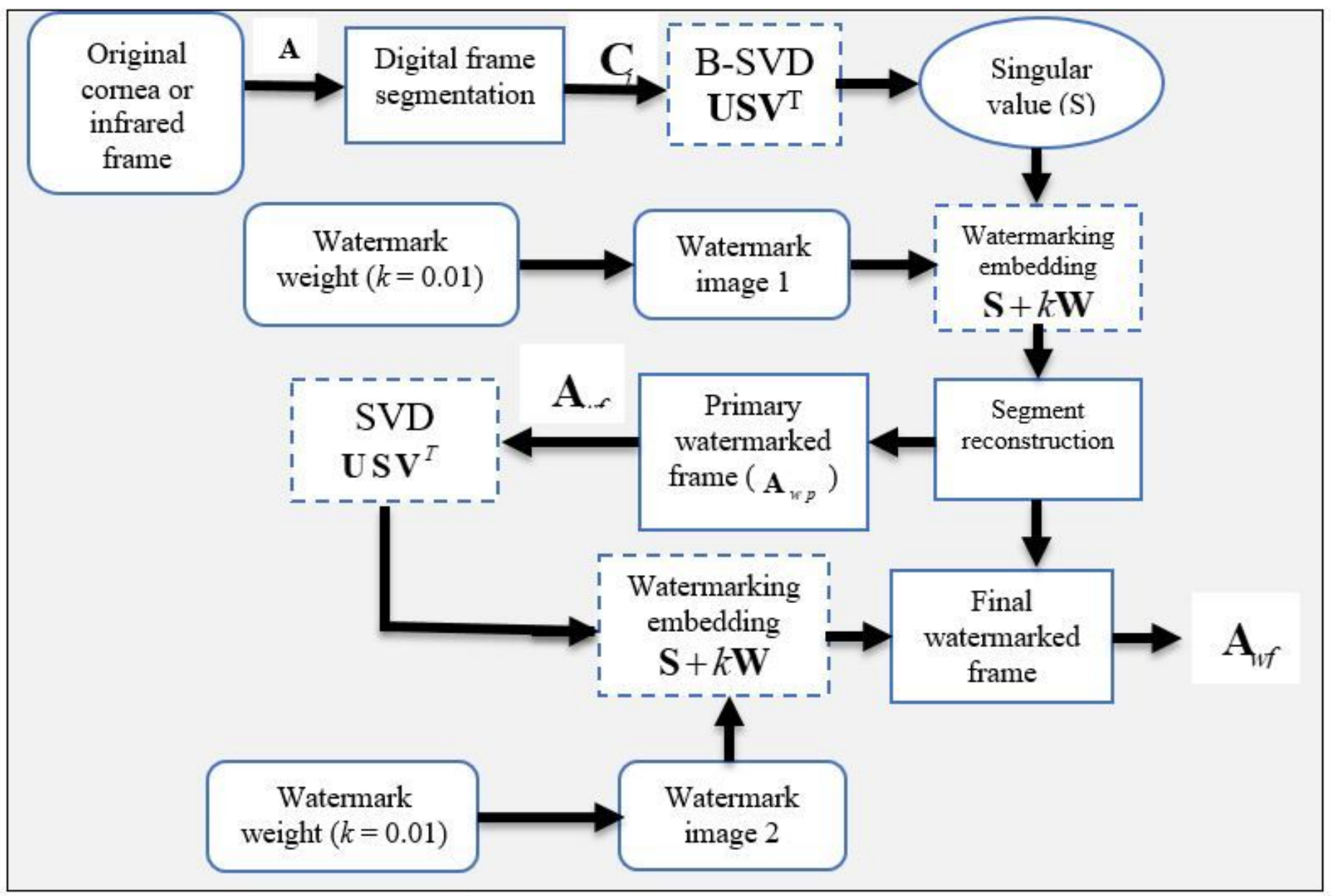

Figure 7

The detailed steps of the proposed embedding process. 


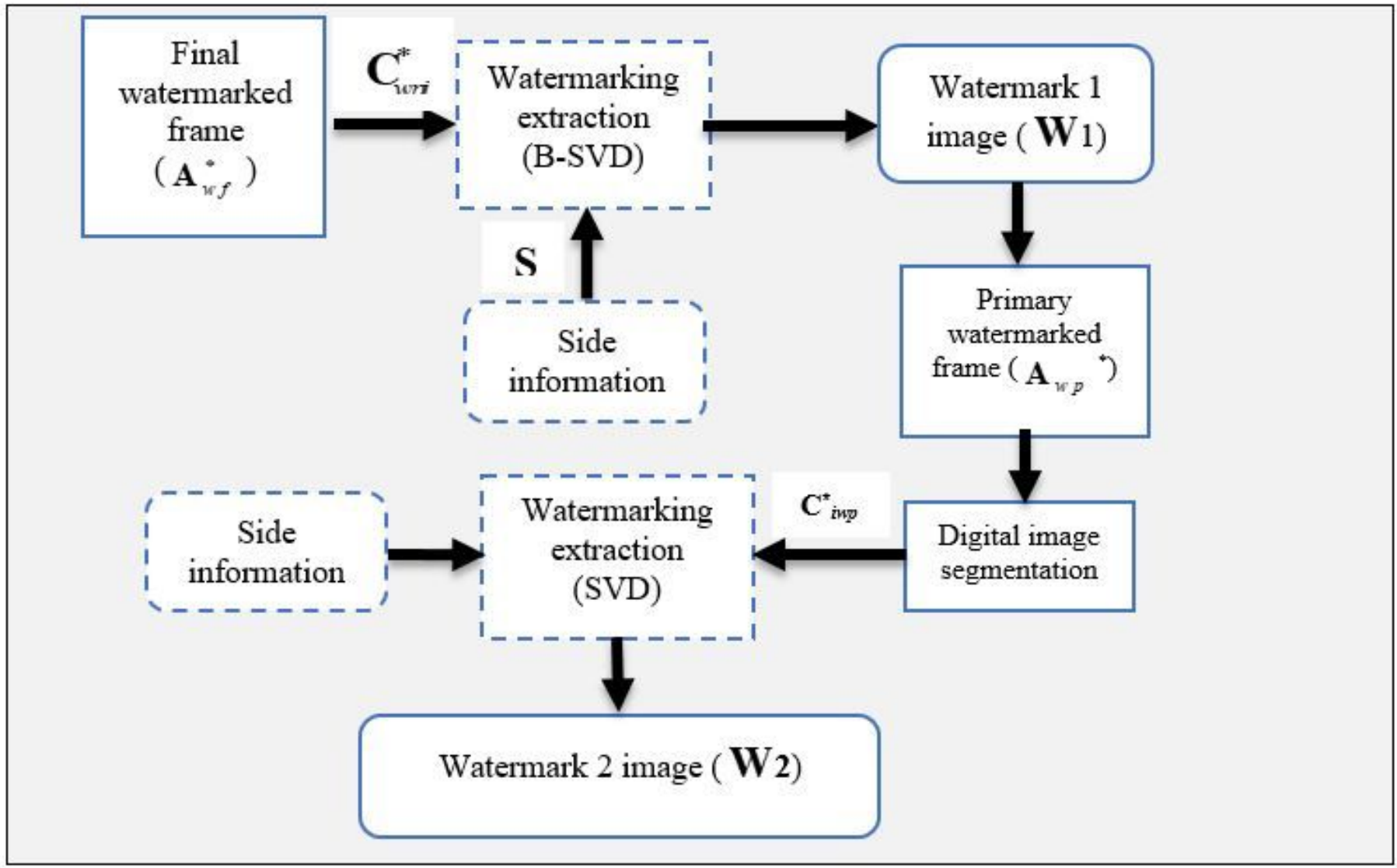

Figure 8

The detail steps of the proposed watermark extraction process. 


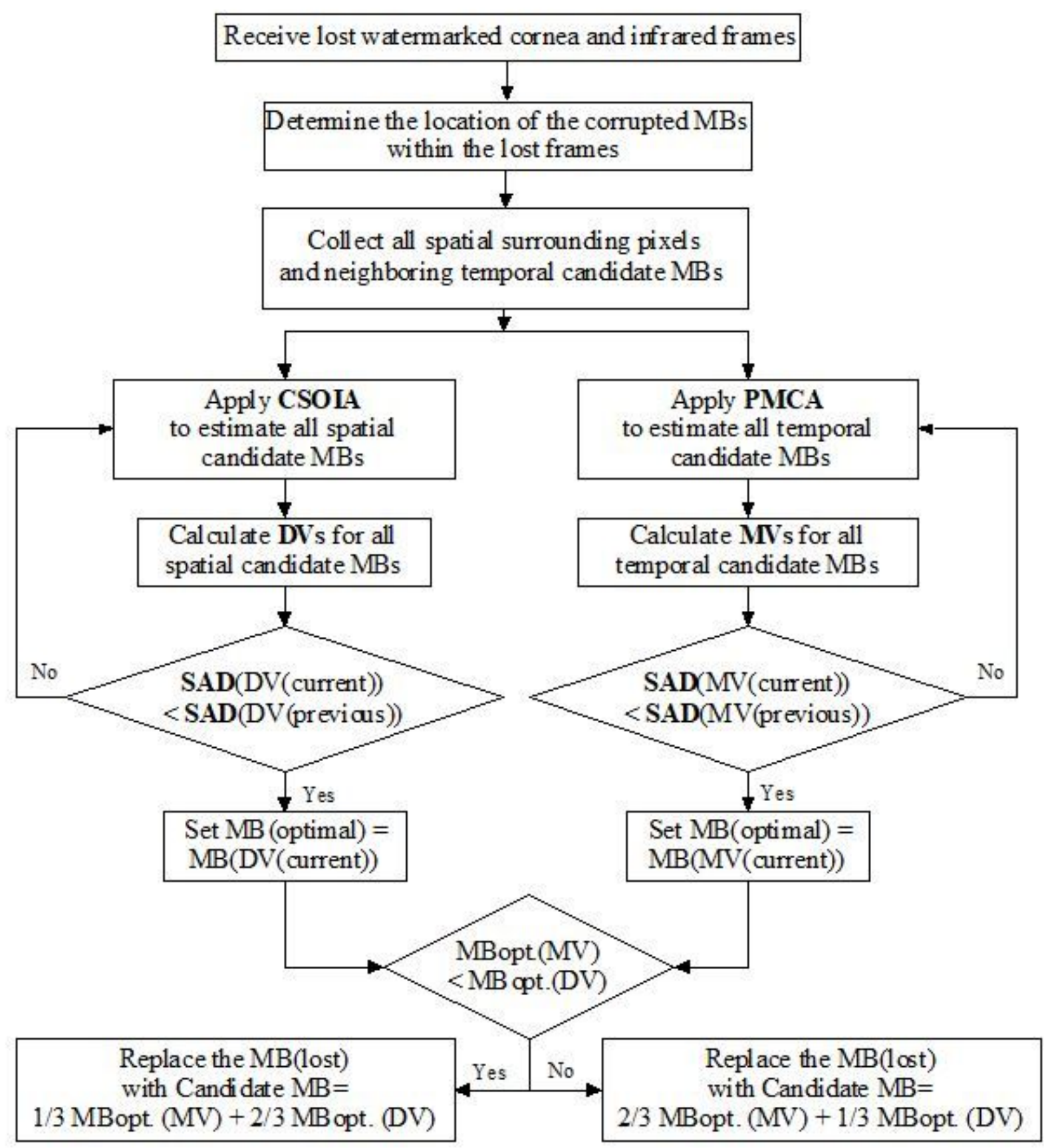

Figure 9

Proposed framework of post-processing error control schemes. 


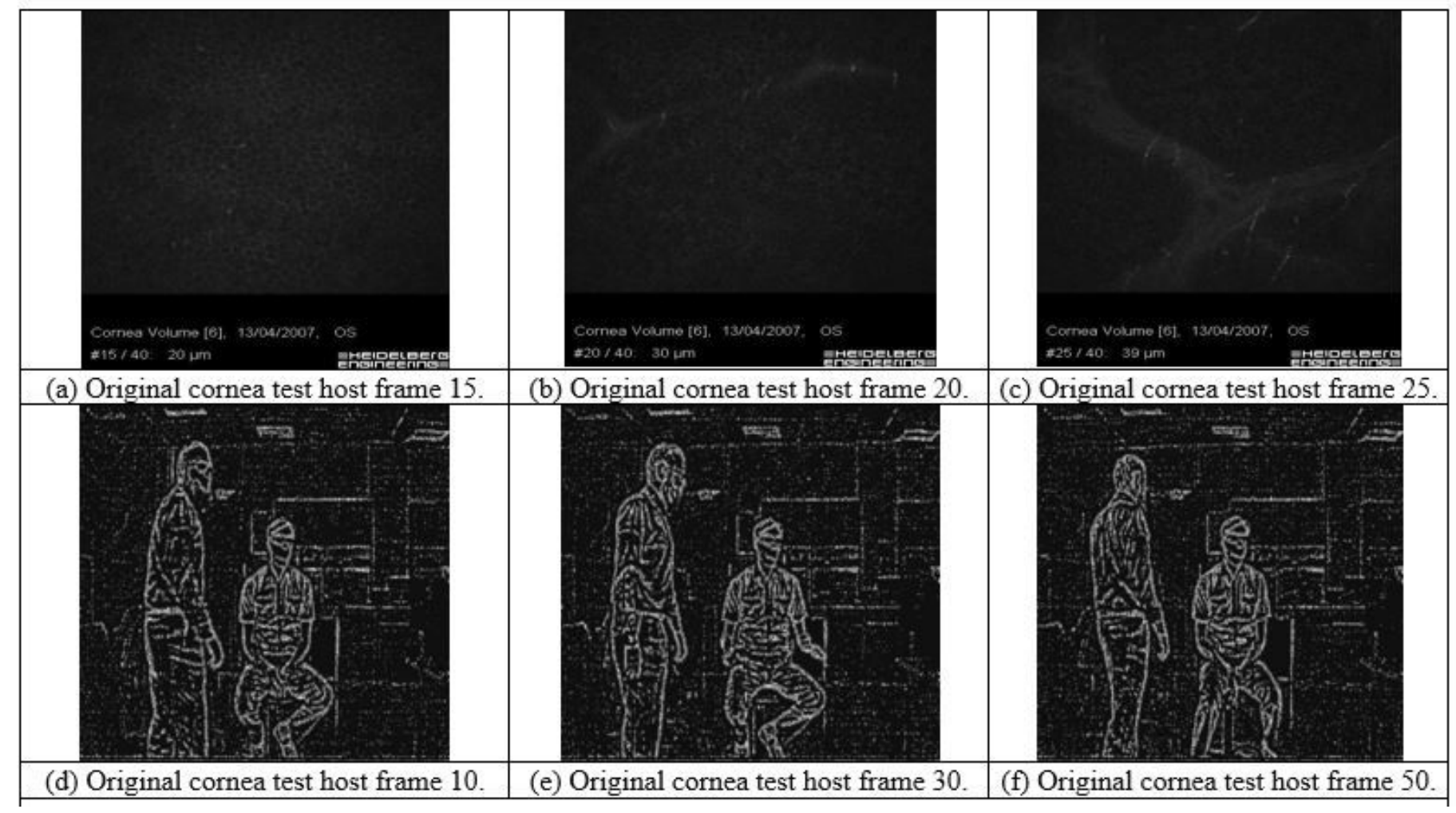

Figure 10

The cornea and infrared tested host frames.

\begin{tabular}{|l|l|}
\hline & \\
\hline (a) Original watermark 1. & (b) Original watermark 2. \\
\hline
\end{tabular}

Figure 11

The original watermark images 1 and 2 . 